\title{
Forward propagation of acoustic pressure pulses in 3D soft biological tissue $\mathbb{I}$
}

\author{
TROND VARSLOT*† and SVEIN-ERIK MÅ $\varnothing Y \ddagger$
}

Keywords: Medical ultrasound, nonlinear acoustics, parabolic approximation, numerical simulation, operator splitting

\begin{abstract}
A simulation method for forward propagation of acoustic pressure pulses in a medium with three-dimensional (3D) spatially-variable acoustic properties is presented. The intended application is to study aspects of ultrasound imaging of soft biological tissue.

The forward wave propagation is modelled by a one-way wave equation. The equation describes tissue exhibiting nonlinear elasticity and arbitrary frequencydependent attenuation.

A numerical solution to the equation is found by means of first-order accurate operator splitting and propagation along the spatial depth coordinate. Thus diffraction, nonlinearity and attenuation are solved independently at each propagation step, rendering their relative importance easy to monitor.

The method is seen to yield an accurate simulation of the wave propagation when compared to numerical solutions of the full wave equation and experiments in a water tank.

By this approach it is possible to simulate wave propagation over relatively large distances - typically several hundred wavelengths - at a modest computational complexity compared to solution of the full wave equation. It furthermore facilitates a high degree of parallelism, thus enabling efficient distribution of the required computations overmultiple processors.
\end{abstract}

\section{Introduction}

The quality of an ultrasound image is limited by the ability to transmit a focused sound beam through the body. The signal received at the transducer is often distorted by multiple reflections, as well as arrival time and amplitude fluctuations caused by variable tissue parameters. The former is known as reverberation, and the latter wavefront aberration. The resolution of an ultrasound image is limited by these factors. Experimental studies of abdominal wall Haberkorn et al. (1993), Hinkelman et al. (1994) and breast tissue Trahey et al. (1991), Freiburger et al. (1992), Zhue \& Steinberg (1994) as well as simulations Tabei et al. (2003), Måsøy et al. (2003) indicate that this aberration can significantly reduce the image resolution. The current paper presents a 3D implementation of a simulation model capable of describing wavefront aberration.

Several authors have performed simulation of ultrasound wave propagation previously Tabei et al. (2003), Måsøy et al. (2003), Nachman et al. (1990), Christopher \& Parker (1991), Berkhoff \& Thijssen (1996), Wojcik et al. (1998), Mast (2002). Depending on their objectives, these simulations range from solving a full wave equation in a heterogeneous medium, to solving an approximate wave equation in a homogeneous medium.

*Corresponding author.

$\dagger$ Department of Mathematical Sciences, Norwegian University of Science and Technology.

$\$$ Department of Circulation and Imaging, Norwegian University of Science and Technology.

IAA early version of this paper was presented at SIMS 2005, the 46th Conference on Simulation and Modeling, Trondheim, Norway, October 13-14, 2005. 
There are several wave equations available for describing acoustic wave propagation Taraldsen (2001), Angelsen (2000), Hamilton \& Blackstock (1997). These equations are most commonly solved by propagation in time, and describe both aberration and reverberation. However, when propagating over large distances, such methods are expensive in terms of memory and computational costs Wojcik et al. (1998), Mast (2002). This is the major reason why most of the simulation-based investigations of ultrasound wave propagation in heterogeneous media have been conducted in 2D.

The current objective is to facilitate studies of the wavefront aberration. This is a phenomenon which is related to the forward-propagation of the pulse. Furthermore, reverberations have been reported to produce only minor distortions of the forward propagating pulse in soft tissue Angelsen (2000), Hamilton \& Blackstock (1997), Mast et al. (1998). For this reason it is important to accurately model forward propagation, but of lesser importance to model multiple reflections.

A parabolic approximation ("the $15^{\circ}$ approximation") of the wave equation is often used in the context of focused (directional) sound beams Tappert (1977). This leads to the Khokhlov-Zabotskaya-Kuznetsov (KZK) equation Zabotskaya \& Khoklov (1969), Kuznetsov (1971), or variations of it. The KZK equation is conveniently solved using operator splitting and propagation in space. Wide-angle parabolic approximations are also available. These are frequently used in underwater acoustics and geophysical applications, and lead to higher-order partial differential equations Claerbout (1976). They do not, however, appear that frequently in ultrasonic imaging, where the $15^{\circ}$ approximation is thought to be adequate.

The KZK-equation is a one-way wave equation in the sense that it only models forward propagation. As a result the computational complexity of solving this equation is much lower than that of solving the full wave equation. Fast numerical solutions may therefore be implemented Hamilton \& Blackstock (1997). Combined with reasonable boundary conditions such as a perfectly matched layer (PML), Berenger (1994), Collino (1997), the equation accurately models the propagation of focused ultrasound beams in a homogeneous medium Bouakaz (2003). In a heterogeneous medium, however, where reflections are important, the KZK equation will not provide an accurate description. This is the case for a medium containing bone structures surrounded by muscle and fat.

Presented here is a one-way wave equation for propagation of ultrasound in heterogeneous soft tissue. A numerical solution of this equation is then found by means of operator splitting. The work is related to an extension of the parabolic approximation to heterogeneous media Bamberg et al. (1988).

This simulation model has previously been presented in the context of $2 \mathrm{D}$ simulations Varslot \& Taraldsen (2005), Varslot (2004). However, in order to keep the paper self-contained, important points are repeated here. A derivation of the underlying wave equation is also included in order to make available to the reader the approximations made in the modelling.

\section{Theory}

\subsection{Modelling}

Let $r$ be the equilibrium position in space of a point-particle, and $r_{\mathrm{E}}$ the position of that same particle at time $t$. Define a function $\Psi(r, t)$ as

$$
r_{\mathrm{E}}(r, t)=r+\Psi(r, t)
$$

This function describes the movement in space of the point-particle. A point-particle is referred to as a material point, and $r$ is the Lagrange coordinate or material coordinate 
of the material point. The function $\Psi$ thus relates the Lagrange coordinate to the Euler coordinate $r_{\mathrm{E}}$. For the deformations considered here, the function $\Psi$ is invertible and differentiable with respect to both $t$ and $r$.

The velocity of the material point is naturally defined as

$$
v(r, t)=\frac{\partial r_{\mathrm{E}}(r, t)}{\partial t}=\frac{\partial \Psi(r, t)}{\partial t} .
$$

Equation (1) specifies a transformation from Lagrangian coordinates to Euler coordinates. Associated with this transformation is the deformation gradient tensor

$$
F=I+\frac{\partial \Psi}{\partial r}=\left(\begin{array}{rrr}
1+\frac{\partial \Psi_{1}}{\partial r_{1}} & \frac{\partial \Psi_{1}}{\partial r_{2}} & \frac{\partial \Psi_{1}}{\partial r_{3}} \\
\frac{\partial \Psi_{2}}{\partial r_{1}} & 1+\frac{\partial \Psi_{2}}{\partial r_{2}} & \frac{\partial \Psi_{2}}{\partial r_{3}} \\
\frac{\partial \Psi_{3}}{\partial r_{1}} & \frac{\partial \Psi_{3}}{\partial r_{2}} & 1+\frac{\partial \Psi_{3}}{\partial r_{3}}
\end{array}\right)
$$

and the Jacobian of the transformation

$$
|F| \equiv \operatorname{det} F \text {. }
$$

Of interest here is the situation where particle movement is confined to small vibrations about the equilibrium position as a result of stretching and compression. The mass density, $\rho$, will consequently be time-dependent. However, conservation of mass may be used to obtain a simple expression for this time-dependence. Let $V_{0}$ be a region in space. Let $V(t)$ be a region in space such that

$$
\left[r \in V_{0}\right] \Leftrightarrow[r+\Psi(r, t) \in V(t)] .
$$

Let the mass density at equilibrium be $\rho_{0}(r)$. Since the same particles are contained in $V_{0}$ and $V(t)$, conservation of mass implies that

$$
\int_{V_{0}} \rho_{0}(r) \mathrm{d} r=\int_{V(t)} \rho\left(r_{\mathrm{E}}, t\right) \mathrm{d} r_{\mathrm{E}}=\int_{V_{0}} \rho(r, t)|F| \mathrm{d} r .
$$

Therefore, $\rho_{0}(r)=\rho(r, t)|F|$ whenever $\rho$ is continuous with respect to $r$.

Shear forces give rise to shear waves that travel at only $1 / 10$ of the speed of pressure waves in soft tissue. These are therefore negligible, and only the acoustic pressure contributes as a net force acting on the medium. If $p$ is the acoustic pressure and $\nabla_{\mathrm{E}}$ denotes the gradient with respect to Euler coordinate $r_{\mathrm{E}}$, then the acoustic pressure forces in Lagrangian coordinates are given by a change of variables

$$
-\int_{V_{t}} \nabla_{\mathrm{E}} p \mathrm{~d} r_{\mathrm{E}}=-\int_{V_{0}}\left(F^{-1}\right)^{T} \nabla p|F| \mathrm{d} r .
$$

Using Equation (2), the momentum for a given control volume $V_{0}$ is

$$
\mathrm{p}\left(V_{0}\right)=\int_{V_{0}} \rho \frac{\partial \Psi}{\partial t}|F| \mathrm{d} r=\int_{V_{0}} \rho_{0} \frac{\partial \Psi}{\partial t} \mathrm{~d} r .
$$

Combining this with Equation (3), conservation of momentum implies that

$$
\rho_{0} \frac{\partial^{2} \Psi}{\partial t^{2}}=-|F|\left(F^{-1}\right)^{T} \nabla p
$$

Since there are four unknown quantities: $p, \Psi_{1}, \Psi_{2}$, and $\Psi_{3}$, another equation is needed 
for the problem to be well-posed. The fourth equation will in this instance be specified as a constitutive relation between the pressure and the density.

A second-order Taylor expansion of the pressure-density relation is commonly used,

$$
p(\rho)=A\left(\frac{\rho-\rho_{0}}{\rho_{0}}\right)+\frac{B}{2}\left(\frac{\rho-\rho_{0}}{\rho_{0}}\right)^{2} .
$$

By conservation of mass, the density is removed in favour of $|F|$

$$
p(|F|)=A\left(\frac{1-|F|}{|F|}\right)+\frac{B}{2}\left(\frac{1-|F|}{|F|}\right)^{2} .
$$

Solving for $1-|F|$ and retaining terms up to $p^{2}$ yields

$$
1-|F|=\kappa p-\beta_{n}(\kappa p)^{2},
$$

where $\kappa=1 / A$ is the compressibility and $\beta_{n}=1+B / 2 A$ is the coefficient of nonlinearity Hamilton \& Blackstock (1997). Note that this expansion is performed for constant entropy, that is, no effects related to temperature changes or viscosity are taken into account.

Attenuation caused by heat conduction and viscosity is modelled by a linear operator $\mathcal{L}$

$$
1-|F|=\kappa p-\beta_{n}(\kappa p)^{2}-\kappa \mathcal{L} p .
$$

\subsection{Approximations and scaling}

In Angelsen (2000) [pp. 12.9] it is shown that a good approximation for $|F|$ is

$$
|F| \approx 1+\nabla \cdot \Psi \text {. }
$$

If this approximation is applied to Equation (5), the resulting equation is a nonlinear elasticity relation

$$
-\nabla \cdot \Psi=\kappa p-\beta_{n}(\kappa p)^{2}-\kappa \mathcal{L} p .
$$

For plane waves the simplification $-|F|\left(F^{-1}\right)^{T} \nabla p=\nabla p$ is possible. This is also a good approximation when the radius of curvature of the wave front is large compared to the displacement, as is often the case in medical ultrasound imaging Angelsen (2000). This approximation, inserted into Equations (4) and (7) yields a generalised Westervelt equation Taraldsen (2001) for the acoustic pressure

$$
\kappa \ddot{p}-\nabla \cdot\left(\frac{1}{\rho} \nabla p\right)=\frac{\mathrm{d}^{2}}{\mathrm{~d} t^{2}}\left(\beta_{n} \kappa^{2} p^{2}+\kappa\llcorner p) .\right.
$$

Assuming smooth density variations, a normalised pressure, $p=p_{*} / \sqrt{\rho}$, the following simplification is possible: Brekhovskikh \& Godin (1999)

$$
\nabla \cdot\left(\frac{1}{\rho} \nabla p_{*}\right)=\frac{1}{\sqrt{\rho}} \nabla^{2} p-p \nabla^{2} \frac{1}{\sqrt{\rho}} .
$$

Using this identity together with $\kappa \rho=1 / c^{2}$, a wave equation for the normalised pressure $p$ is obtained,

$$
\nabla^{2} p-\frac{1}{c^{2}} \ddot{p}=g p-\frac{\beta_{n}}{\sqrt{\rho} c^{4}} \frac{\partial^{2} p^{2}}{\partial t^{2}}-\frac{1}{c^{2}} \frac{\partial^{2} \mathcal{L} p}{\partial t^{2}},
$$

where $g=\sqrt{\rho} \nabla^{2}(1 / \sqrt{\rho})$ describes density fluctuations. 
Typical values for tissue parameters are listed in Table 1. Furthermore, considering ultrasound pulses with frequency in the $\mathrm{MHz}$ range and acoustic pressures around $1 \mathrm{MPa}$, a set of natural scales for the equation may be inferred. These scales are listed in Table 2.

\subsection{Approximations}

With an appropriate choice of scale for the speed of sound, the average speed of sound may be assumed to be 1 . Let the deviation from this average be described using $c_{1}(r)$ through

$$
\frac{1}{c^{2}}=1-2 \gamma c_{1}
$$

A suitable value for the dimensionless scaling factor $\gamma$ is 0.1 for soft tissue.

If the main direction of propagation is the $z$-direction, then a change of variables $\tau=t-z$ yields the equation in dimensionless form

$$
\frac{\partial^{2} p}{\partial \tau \partial z}=\frac{1}{2}\left(\nabla^{2}-g\right) p-\varepsilon_{t} \ddot{p}+\frac{\varepsilon_{n}}{2} \frac{\partial^{2} p^{2}}{\partial \tau^{2}}+\varepsilon \frac{\partial^{2} L p}{\partial \tau^{2}} .
$$

The coefficients $\varepsilon_{t}=\gamma c_{1}, \varepsilon_{n}=p_{s} \beta_{n} / \rho_{s} c_{s}^{2} \sqrt{\rho} c^{4}$ and $\varepsilon$ are spatially variable. Values for the coefficients $\varepsilon_{t}, \varepsilon_{n}$ and $\varepsilon$ for different tissue types are given in Table 3 . With the introduction of $\varepsilon$, a convenient change from $\mathcal{L}$ to $L$ has also been made as $\varepsilon L=\mathcal{L} / 2 c^{2}$.

The acoustic pressure, $p_{*}$, may be recovered from the scaled normalised pressure, $p$, through the relation

$$
p_{*}=p_{s} p \sqrt{\rho} \text {. }
$$

Table 1. Values for some physical parameters in medical ultrasound imaging at $1 \mathrm{MHz}$ and $37^{\circ} \mathrm{C}$. (See Duck (1990))

\begin{tabular}{lcccll}
\hline tissue & $\begin{array}{c}c \\
{[\mathrm{~mm} / \mu \mathrm{s}]}\end{array}$ & $\begin{array}{c}\rho \\
{\left[\mathrm{mg} / \mathrm{mm}^{3}\right]}\end{array}$ & $\beta_{n}$ & $\begin{array}{c}\alpha \\
{[\mathrm{dB} / \mathrm{mm}]}\end{array}$ & $b$ \\
\hline fat & 1.436 & 0.928 & 5.8 & 0.30 & 0.9 \\
muscle & 1.550 & 1.060 & 3.9 & 0.05 & 1.1 \\
blood & 1.584 & 1.060 & 4.0 & 0.01 & 1.2 \\
water & 1.524 & 0.993 & 3.7 & 0.00014 & 2.0 \\
\hline
\end{tabular}

Table 2. Scales relating dimensional variables to dimensionless variables.

\begin{tabular}{lll}
\hline speed of sound & $c=c * / c_{s}$ & $c_{s}=1.54 \mathrm{~mm} / \mu \mathrm{s}$ \\
density & $\rho=\rho_{*} / \rho_{s}$ & $\rho_{s}=1 \mathrm{mg} / \mathrm{mm}^{3}$ \\
acoustic pressure & $p=p_{*} / p_{s}$ & $p_{s}=1 \mathrm{MPa}$ \\
time & $t=t_{*} / t_{s}$ & $t_{s}=1 \mu \mathrm{s}$ \\
space & $x=x_{*} / c_{s} t_{s}$ & \\
normalised pressure & $p=p_{*} \sqrt{\rho_{s}} / p_{s}$ & \\
density fluctuation & $g=g_{*}^{2} x_{s}^{2}$ & \\
\hline
\end{tabular}


Table 3. Typical values for the coefficients in Equation (9) at $37^{\circ} \mathrm{C}$.

\begin{tabular}{lrcl}
\hline tissue & $\varepsilon_{t}\left[10^{-1}\right]$ & $\varepsilon_{n}\left[10^{-3}\right]$ & $\varepsilon\left[10^{-3}\right]$ \\
\hline fat & -0.75 & 3.36 & 6.6 \\
muscle & 0.06 & 1.55 & 0.79 \\
blood & 0.27 & 1.46 & 0.15 \\
water & -0.11 & 1.63 & 0.0004 \\
\hline
\end{tabular}

Employing the parabolic approximation $\partial^{2} p / \partial z^{2}=0$, which is valid for directional sound beams, and letting $\nabla^{2}=\nabla_{\perp}^{2}+\partial^{2} / \partial z^{2}$, leads to

$$
\frac{\partial^{2} p}{\partial \tau \partial z}=\frac{1}{2}\left(\nabla_{\perp}^{2}-g\right) p-\varepsilon_{t} \ddot{p}+\frac{\varepsilon_{n}}{2} \frac{\partial^{2} p^{2}}{\partial \tau^{2}}+\varepsilon \frac{\partial^{2} L p}{\partial \tau^{2}} .
$$

For a homogeneous medium (i.e. $g=0$ ) and classical loss $\varepsilon L=\delta \dot{p} / c^{2}$, this is the KZK equation where $\delta$ is the diffusivity Hamilton \& Blackstock (1997).

Integrating Equation (8) with respect to time leads to the final dimensionless equation

$$
\frac{\partial p}{\partial z}=\frac{1}{2} \int_{-\infty}^{\tau}\left(\nabla_{\perp}^{2}-g\right) p \mathrm{~d} \tau+\left(\varepsilon_{n} p-\varepsilon_{t}\right) \dot{p}+\varepsilon \frac{\partial L p}{\partial \tau} .
$$

Equation (9) combined with the boundary condition $p(z=0)=p_{0}(x, y, t)$ uniquely determines the pressure field at a plane $z>0$.

By employing the parabolic approximation, the equation is no longer able to describe waves which travel in the negative $z$-direction. However, as the reflection coefficient between different types of soft tissue is low $(<0.1)$, and reverberations have been reported to produce only minor distortions of the forward propagating pulse in soft tissue, Angelsen (2000), Mast et al. (1998) this should not reduce the accuracy of the simulation of the forward-propagation significantly.

\subsection{Operator splitting}

A mathematical foundation for operator splitting is found by combining the Lie-Trotter product formula Kappel (1997) (Thm. 10.17) with the product integral Dollard \& Friedman (1979).

Equation (9) is of the form

$$
\frac{\partial p}{\partial z}=\left(A_{d}+A_{n}+A_{l}\right) p
$$

where the operators $A_{d}, A_{n}$ and $A_{l}$ account for diffraction and scattering, nonlinear elasticity, and energy loss, respectively

$$
\begin{aligned}
& A_{d}(z) p=\frac{1}{2} \int_{-\infty}^{\tau}\left[\nabla_{\perp}^{2}-g(z)\right] p \mathrm{~d} \tau, \\
& A_{n}(z) p=\left[\varepsilon_{n}(z) p-\varepsilon_{t}(z)\right] \dot{p}, \\
& A_{l}(z) p=\varepsilon(z) \frac{\partial L(z) p}{\partial \tau} .
\end{aligned}
$$


The exponential function $\exp (h A)$ is used to formally denote the operator which sends the initial condition $u(0)$ onto the solution $u(h)$ of the differential equation $\partial u / \partial t=A u$. In this notation, the solution of Equation (9) is denoted $p(z+h)=\exp \left(h\left[A_{d}+A_{n}+A_{l}\right]\right) p(z)$. Furthermore, the formal order of the error for the approximation

$$
p(z+h) \approx e^{h A_{d}} e^{h A_{l}} e^{h A_{n}} p(z)
$$

is $O\left(h^{2}\right)$. This is therefore referred to as a first-order approximation, often denoted as Gudonov splitting. Strang splitting Strang (1968) may be used as an alternative method for combining the solution operators in order to increase the formal order of the approximation, e.g.

$$
p(z+h) \approx e^{\frac{h}{2} A_{d}} e^{\frac{h}{2} A_{n}} e^{h A_{l}} e^{\frac{h}{2} A_{n}} e^{\frac{h}{2} A_{d}} p(z)
$$

The order of convergence, however, will depend heavily on the solution, and not necessarily adhere to this formal order.

\section{Implementation}

The model equation for forward wave propagation is written in the form of an evolution equation. The computation starts at the plane $z=0$ with an initial condition $p(x, y, 0, t)=p_{0}(x, y, t)$. The propagation is performed in steps of length $h$ in the direction of $z$, such that $z_{k}=k h$.

The numerical approximation of the exact solution operator, $\exp (h A)$, is denoted $u_{A}^{h}$. In this notation an approximate solution to the equation as a whole is given by

$$
p\left(z_{k+1}, t\right)=\mathcal{U}_{A_{d}}^{h}\left(z_{k}\right) \mathcal{U}_{A_{n}}^{h}\left(z_{k}\right) \mathcal{U}_{A_{l}}^{h}\left(z_{k}\right) p\left(z_{k}, t\right) .
$$

For the exact solution operators, an arbitrarily accurate approximation may be obtained by choosing a small enough step size to eliminate the splitting error. For the numerical solution, the step size should not be chosen in an arbitrary manner. When the splitting error is of the same order of magnitude as the numerical error in each of the numerical solution operators, decreasing the step size further may, in fact, amplify the error. The step size should ideally be selected such that the splitting error is of the same order of magnitude as the accuracy of each of the numerical solution operators. This may be viewed as a form of Morzov's discrepancy principle known from the theory of regularisation and inverse problems Engl et al. (2000).

\subsection{Absorption}

A phenomenologic model for frequency-dependent absorption which is often used is the power-law absorption model. In particular it provides a good description of soft tissue Duck (1990). Employing this model, the quantity $\varepsilon \partial L p / \partial \tau$ in Equation (9) may be defined through its temporal Fourier transform

$$
\begin{aligned}
\mathcal{F}\{\partial L p / \partial \tau\} & =-|\omega|^{b} \mathcal{F}\{p\}, \\
\varepsilon & =\frac{\ln 10}{20} \frac{a}{(2 \pi)^{b}},
\end{aligned}
$$

where $a$ and $b$ are appropriate tissue-dependent parameters. The operator $L$ as defined by 
Equation (13) violates the causality principle. As such the model is not physically correct. It may however be amended by letting

$$
\mathcal{F}\{\partial L p / \partial \tau\}=\left[-|\omega|^{b}+i \Delta(\omega)\right] \mathcal{F}\{p\} .
$$

Causality implies that $\Delta(\omega)$ should be the Hilbert transform of $|\omega|^{b}$ Szabo (1994).

Due to the spectral definition of the absorption, the Fourier transform is well suited to define a solution operator for the absorption term. Letting $\mathcal{F}$ and $\mathcal{F}^{-1}$ be the temporal Fourier transform and its inverse transform, respectively,

$$
\begin{aligned}
p\left(z_{k+1}, \tau\right) & =e^{h A_{l}\left(z_{k}\right)} p\left(z_{k}, \tau\right) \\
& =\mathcal{F}^{-1}\left[\mathcal{F}(p)\left(z_{k}, \omega\right) \exp \left(-\varepsilon\left(z_{k}\right) H\left(z_{k}, \omega\right) h\right)\right],
\end{aligned}
$$

with

$$
H\left(z_{k}, \omega\right)=(1-i \mathcal{H})|\omega|^{b\left(z_{k}\right)},
$$

and $\mathcal{H}$ denoting the Hilbert transform. Using the Fast Fourier Transform (FFT) in the implementation, a solution operator $\mathcal{U}_{A_{l}}^{h}\left(z_{k}\right)$ is obtained.

The main limitation for the accuracy of this solution operator is in applying the FFT over discontinuities at the edges of the signal. The computation domain is therefore large enough in the temporal direction to make the pulse taper to zero at both ends.

\subsection{Nonlinearity}

When the step size $h$ is short, i.e. $h<\operatorname{supp} 1 /\left|\partial p / \partial z\left(z_{k}, \tau\right)\right|$, the nonlinear term is solved by the method of characteristics

$$
\begin{aligned}
p\left(z_{k+1}, \tau\right) & =p\left(z_{k}, \tau_{k}-h \Delta\left[z_{k}, p\left(z_{k}, \tau_{k}\right)\right]\right), \\
\Delta\left[z_{k}, p\left(z_{k}, \tau_{k}\right)\right] & =\frac{\varepsilon_{n}\left(z_{k}\right)+\varepsilon_{n}\left(z_{k+1}\right)}{2} p\left(z_{k}, \tau_{k}\right)-\frac{\varepsilon_{t}\left(z_{k}\right)+\varepsilon_{t}\left(z_{k+1}\right)}{2} .
\end{aligned}
$$

This defines the solution at grid points which are not equally spaced in the temporal direction. In order to preserve equally-spaced grid points, the function $p\left(z_{k+1}, t\right)$ is resampled. This introduces an interpolation error. As long as the pulse is sampled with a sufficiently high sampling frequency, the interpolation error is negligible. The solution operator including the re-sampling is $u_{A_{n}}^{h}\left(z_{k}\right)$.

There is, of course, a potential problem here in the event of shock formation. If the step size has to be chosen increasingly smaller, then the forward propagation would halt. This could be resolved by a different solution method such as front-tracking Holden \& Risebro (2002). However, absorption in soft tissue is sufficiently large to avoid shock formation for the pressure pulses of interest. Therefore, these problems are avoided by adaptively setting the step size small enough and using a high enough temporal sampling rate.

\subsection{Diffraction: parabolic model}

In order to find a numerical solution for the diffraction and scattering effects defined in Equation (10), an implicit Euler scheme was implemented 


$$
\begin{aligned}
p\left(z_{k+1}, \tau_{k}\right)= & p\left(z_{k}, \tau_{k}\right)+h \frac{\partial p}{\partial z}\left(z_{k+1}, \tau_{k}\right) \\
= & p\left(z_{k}, \tau_{k}\right)+p\left(z_{k+1}, \tau_{k-1}\right)-p\left(z_{k}, \tau_{k-1}\right) \\
& +h \frac{1}{2} \int_{\tau_{k-1}}^{\tau_{k}}\left[\frac{\partial^{2}}{\partial x^{2}}-g\left(z_{k+1}\right)\right] p\left(z_{k+1}, \tau\right) \mathrm{d} \tau .
\end{aligned}
$$

The second derivative of $p$ with respect to $x$ was approximated by a standard fourth-order central differencing scheme which may be represented by a banded matrix $D$. Furthermore, the integral was evaluated using a trapezoidal approximation. Let $I$ denote the identity matrix and $B_{k}=D-\operatorname{diag}\left[g\left(z_{k+1}\right)\right]$, where $\operatorname{diag}\left[g\left(z_{k}\right)\right]$ is the diagonal matrix with entries from $g\left(z_{k}\right)$. Let $h_{\Delta}=h \Delta t / 4$. Then

$$
\left(I-h_{\Delta} B_{k}\right) p\left(z_{k+1}, \tau_{k}\right)=p\left(z, \tau_{k}\right)-p\left(z_{k}, \tau_{k-1}\right)+\left(I+h_{\Delta} B_{k}\right) p\left(z_{k+1}, \tau_{k-1}\right) .
$$

This set of equations may be solved inductively by assuming the solution to be zero for some time $\tau_{0}$.

In a limited computational domain, appropriate boundary conditions must be applied in order to avoid reflection artifacts. This was achieved by adding a PML at the boundary of the domain Berenger (1994), Collino (1997).

\subsection{Diffraction: pseudo-differential model}

Equation (9) is exact for simple waves, and a good approximation for directive sound beams when the curvature of the wave front is small. In a heterogeneous medium, however, the wave front may undergo deformations which cause the curvature to be too large for this approximation to be adequate. Higher-order parabolic approximations may be used to improve the results in such cases Bamberg et al. (1988). Implementation of these is also discussed in Bécache et al. (1998). Alternatively, when $\rho$ is constant, the diffraction operator resulting from the full wave equation may be solved in the forward direction using the angular spectrum method.

Define the function $U$ as

$$
U\left(k_{x}, k_{y}, \omega, h\right)= \begin{cases}e^{-i h \omega\left(\sqrt{1-\left(k_{x}^{2}+k_{y}^{2}\right) / \omega^{2}}-1\right)} & , \omega^{2}>k_{x}^{2}+k_{y}^{2} \\ e^{i h \omega\left(i \sqrt{\left(k_{x}^{2}+k_{y}^{2}\right) / \omega^{2}-1}+1\right)} & , \text { otherwise. }\end{cases}
$$

A solution in the forward direction is then given by

$$
p(x, z+h, \tau)=\mathcal{F}^{-1}\left\{U\left(k_{x}, k_{y}, \omega, h\right) \hat{p}\left(k_{x}, k_{y}, z, \omega\right)\right\},
$$

where $\mathcal{F}$ represents Fourier transform with respect to $x, y$, and $t$. This solution is what Bamberg et al. refer to as approximating the wave equation by a pseudo-differential equation Bamberg et al. (1988). The resulting one-way wave equation will therefore be referred to as the pseudo-differential model. In addition to the boundary condition of $p(z)=p_{0}(x, y, t)$ it will implicitly satisfy an outgoing radiation condition which ensures that the solution propagates in the positive $z$-direction. If $\rho$ is not constant, an approximative solution may be defined in a similar way Varslot (2004).

Employing the FFT in the implementation imposes periodic boundary conditions. For a focused beam, however, adequate suppression of the effect of periodicity is obtained by applying a spatial window-function to the solution at regular intervals, thus tapering the solution to zero at the edges. 


\section{Examples}

The performance of the presented simulation method is demonstrated by its propensity to produce numerical results which compare favourably to various references, including known analytic solutions, measurements in a water tank and to a numerical solution to the full wave equation.

\subsection{Homogeneous tissue}

4.1.1. Burgers equation - analytic In the case of plane waves in water, Equation (9) reduces to

$$
p_{z}=\varepsilon_{n} p \dot{p}+\varepsilon \ddot{p} .
$$

By a simple change of variables $t=z$ and $x=-\varepsilon_{n} \tau$, this may be transformed into a viscous Burgers' equation on standard form with viscosity $v=\varepsilon / \varepsilon_{n}^{2}$. An analytic solution based on a $\delta$-pulse initial condition may therefore be used as a reference in this case Whitham (1974).

Figure 1 shows the relative $L^{2}$-error

$$
\text { err }=\sqrt{\frac{\int\left|p_{\text {num }}-p_{\text {ref }}\right|^{2} \mathrm{~d} t}{\int\left|p_{\text {ref }}\right|^{2} \mathrm{~d} t}}
$$

between the reference solution and the numerical solution. The plot indicates that the Gudonov splitting scheme has a local error of order slightly less than $h^{2}$, almost matching the formal order of the scheme. The Strang splitting has a local error of order somewhere between two and three for this initial condition, and does not, therefore, obtain its formal order of two. The local error of both schemes has a kink where the accuracy changes. When the step size is decreased beyond this point, the local error seems to be of order $h$. The kink indicates the point at which the splitting error becomes insignificant compared to the error of each solution operator. Increasing the individual solution operator accuracy, i.e. increasing the temporal sampling rate, shifts the kink downwards, but at the expense of increased computational and memory cost. The dashed line clearly demonstrates this as the temporal sampling rate is increased by a factor of two.

4.1.2. Hydrophone measurements A test of the model as well as the numerics was performed using hydrophone measurements recorded in a water tank experiment as a reference. The measurements used in this study were recorded in a water tank using a $0.4 \mathrm{~mm}$ needle hydrophone (Force Instruments MI2225). A pulse with centre frequency of $2.2 \mathrm{MHz}$ was transmitted from a $1.5 \mathrm{D}$ rectangular phased array probe (M3S) with dimensions $22 \mathrm{~mm} \times 13 \mathrm{~mm}$ and focal distance of $70.0 \mathrm{~mm}$. In order to obtain an initial condition for the numerical solution to the wave propagation, measurements of the near-field were recorded $1.0 \mathrm{~mm}$ away from the probe surface, perpendicular to the focal axis (see Figure 2). By doing so, the problem of modelling the physical characteristics of the transducer, for example the element sizes and the acoustic coupling between the transducer and the tissue, was avoided.

The near-field was numerically propagated a distance $69.0 \mathrm{~mm}$ to the focal plane of the probe. Figures 3 and 4 display consistency between the numerical solution of the model and the measurements. Discrepancy should be expected due to factors like calibration of the hydrophone and impurities in the water. 


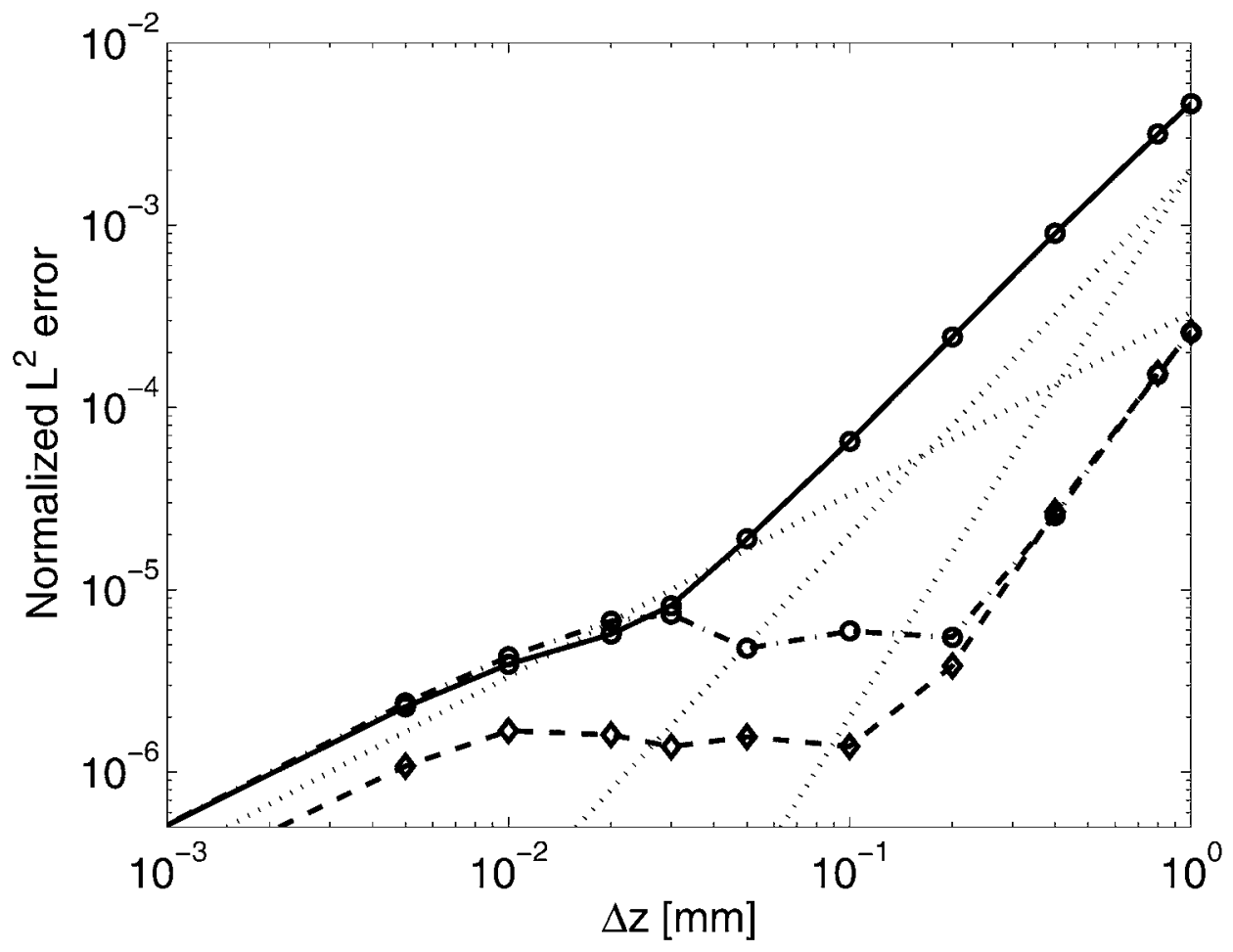

Figure 1. Relative local $L^{2}$ error for the numerical solution when compared to an analytic solution to Burgers' equation. Solid line: Gudonov splitting. Dash-dot line: Strang splitting. Dashed line: Strang splitting with more accurate solution operators. Dotted lines: slopes for local first-, second- and third-order schemes.

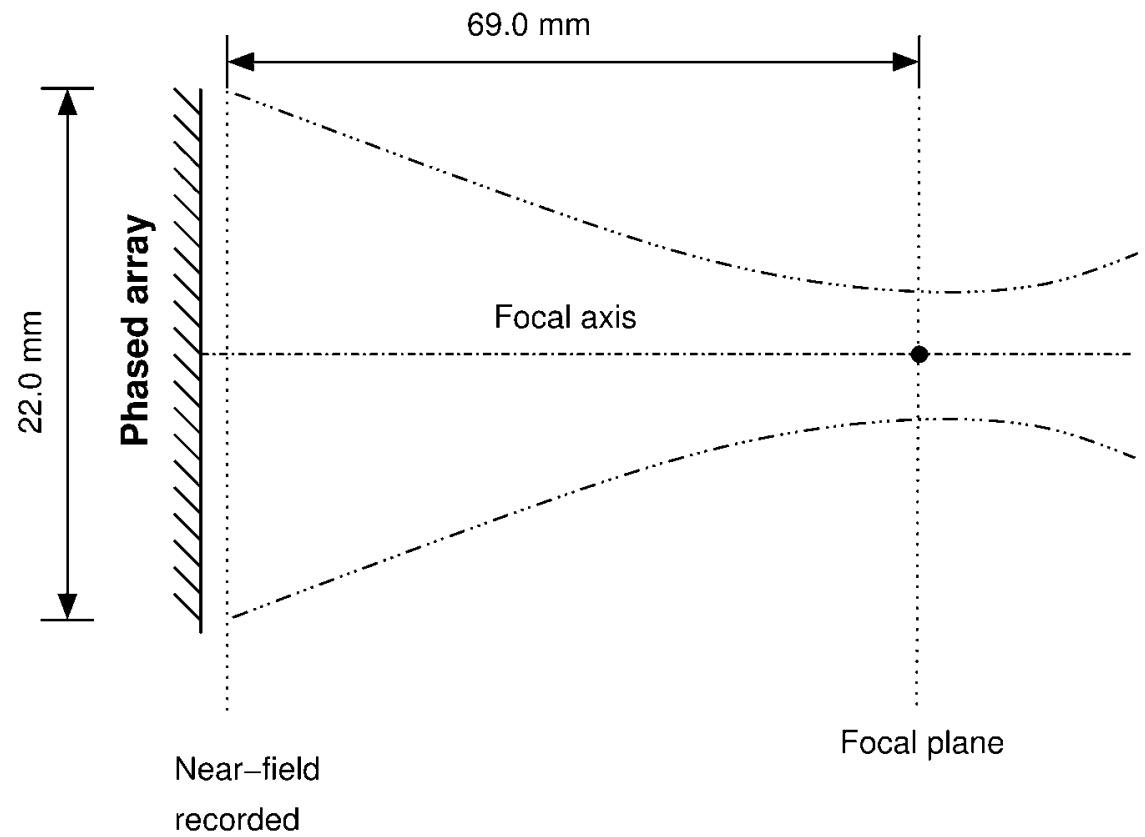

Figure 2. Experimental setup for comparing the KZK-based simulation to hydrophone measurements. 

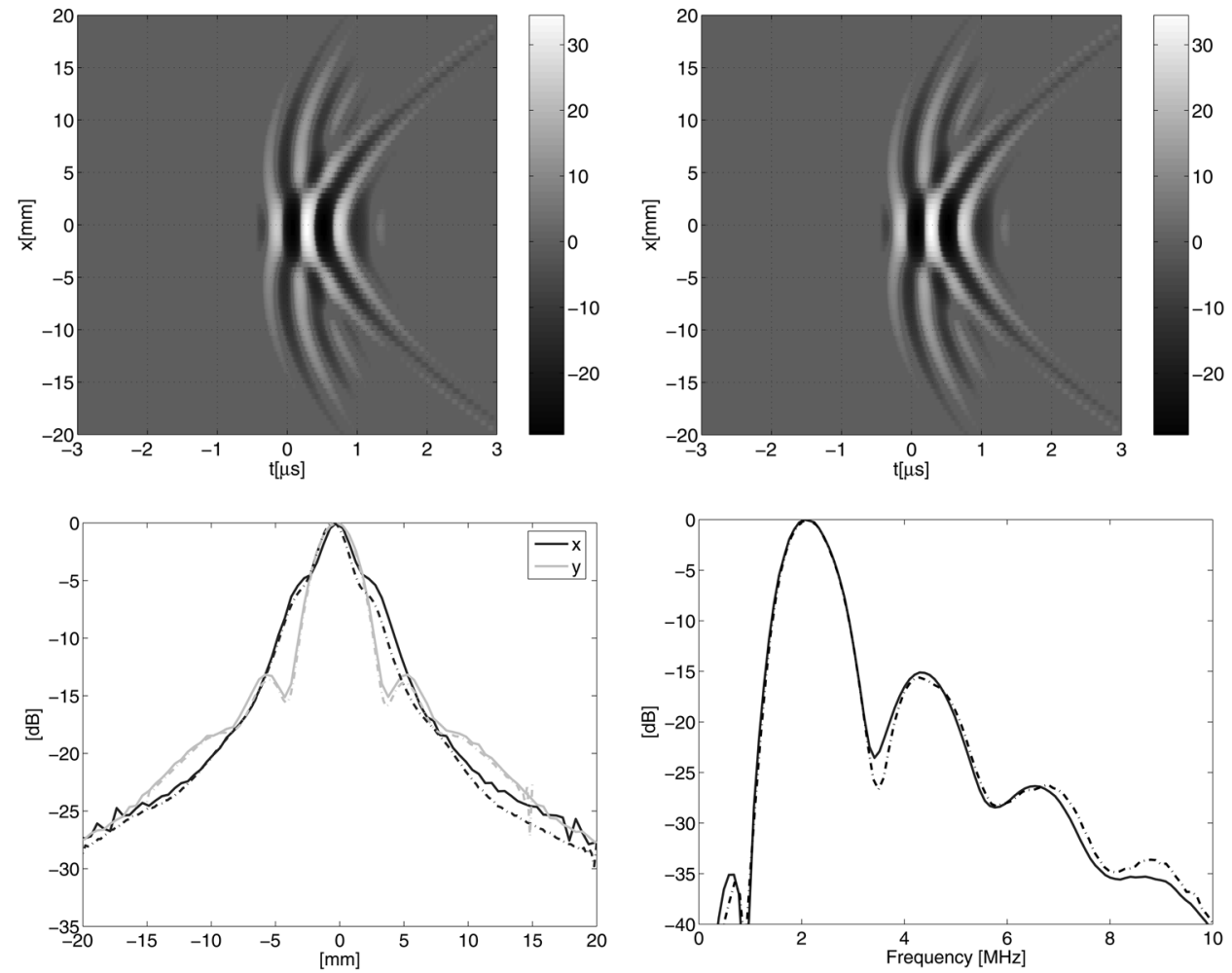

Figure 3. Comparison between measurement and numerical propagation in the focal plane, $69.0 \mathrm{~mm}$ away from the initial plane. Top left: measured acoustic field on the $x$ axis. Top right: numerically propagated acoustic field on the $x$-axis. Bottom left: temporal rms-value for the numerically propagated (solid lines) and measured (dash-dot lines) acoustic fields along $x$-axis and $y$-axis. Bottom right: spectrum of measured and numerically-propagated pulses at the focal point.

\subsection{Heterogeneous tissue}

In order to evaluate how accurately the one-way wave equation approximates the propagation through a heterogeneous medium, a simulation by the presented method was compared to a numerical solution of the full wave equation. A speudo-spectral numerical solution of a comparable wave equation was presented by Wojcik et al. (1997). As there is a publicly-available 2D-implementation of this pseudospectral method Andersen (2000), the comparison was performed for a 2D simulation.

A plane wave propagating in the $z$-direction was used as an initial condition for the full wave equation. The propagating wave was recorded in two planes parallel to the wave front, one at each side of the heterogeneity in the tissue. The recorded incoming wave was then used as the initial condition for the numerical solution of both the parabolic model and the pseudo-differential model. The solutions were then compared with the recorded wave front obtained from the pseudo-spectral solver of the full wave equation.

Figure 5 shows the sound speed variations in the heterogeneous tissue used for the simulation comparison.

Figure 6 displays consistency between the numerical solution of the full wave equation and the numerical solution of the parabolic model. However, it is clear that the 


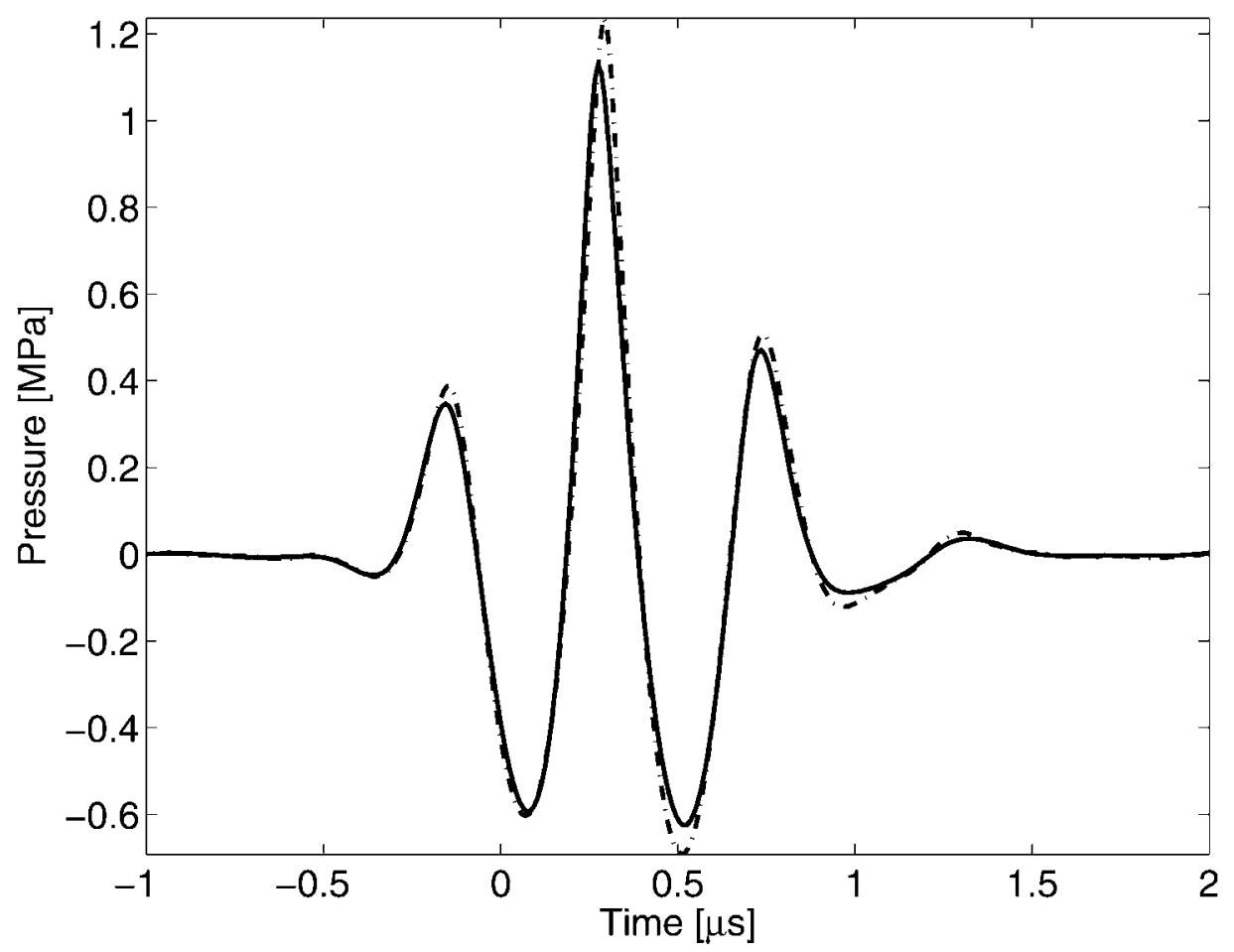

Figure 4. Comparison between the measured and numerically propagated acoustic field at the focal point, $69.0 \mathrm{~mm}$ away from the initial plane.

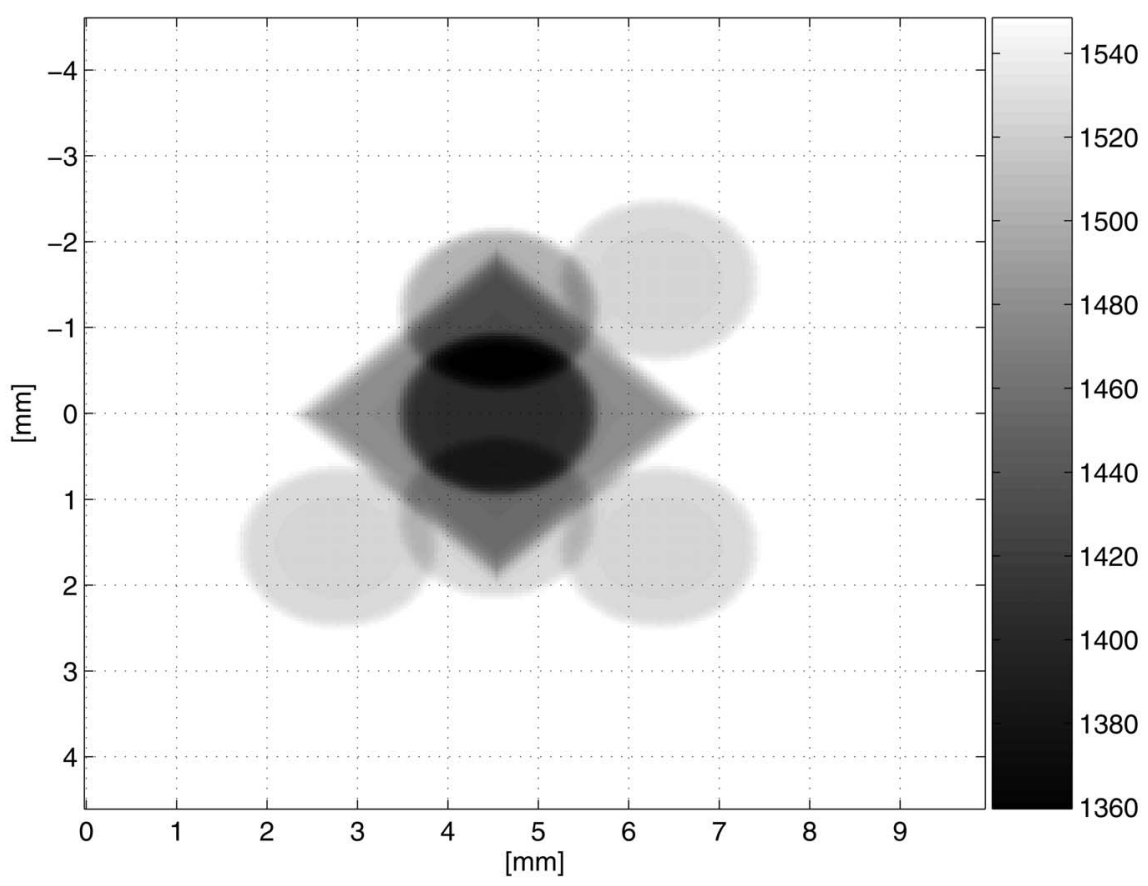

Figure 5. Sound speed variations used for the simulation comparison. 

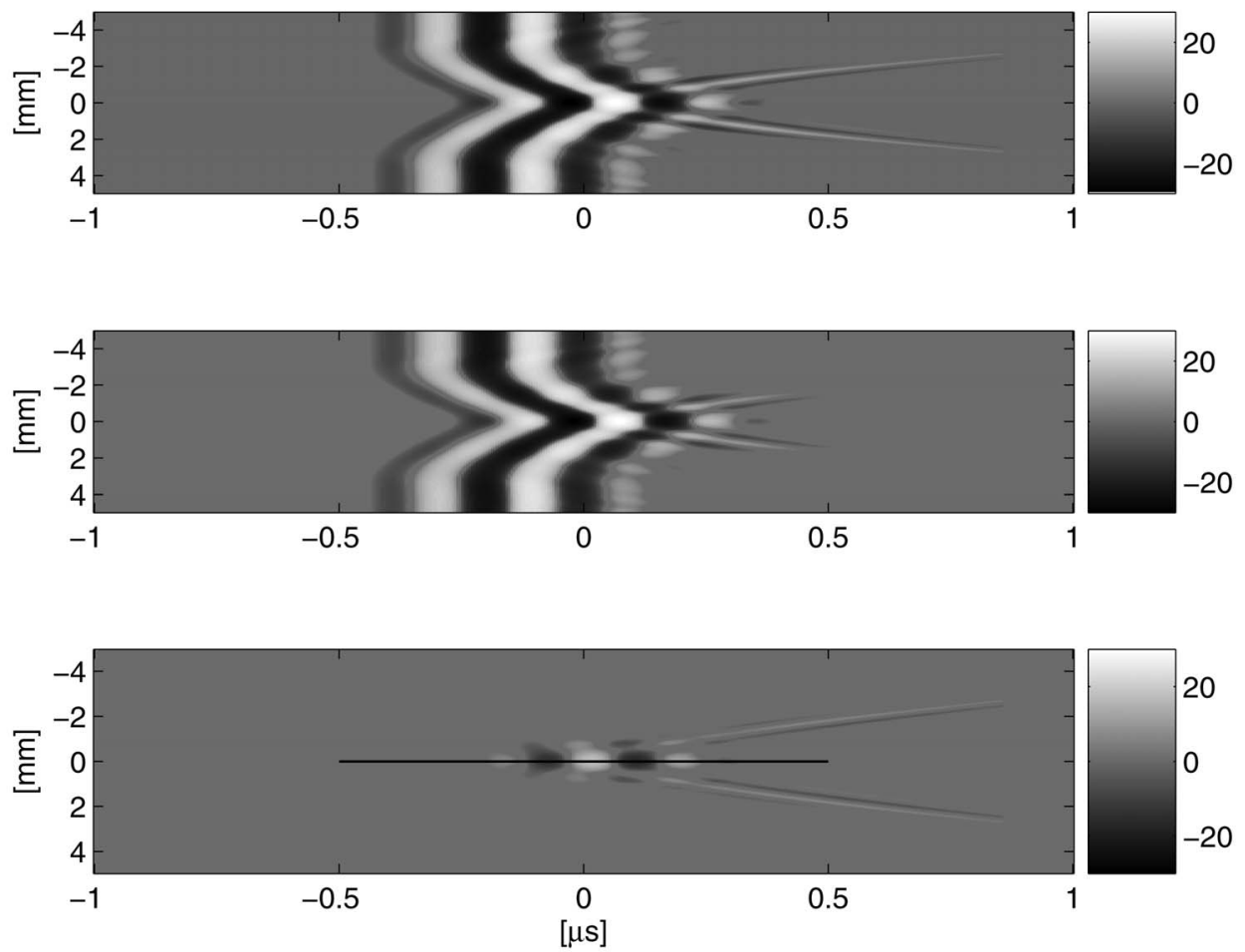

Figure 6. Comparison of the forward propagating wave fronts for the one-way wave equation using the parabolic approximation and the full wave equation. The wave fronts were recorded at a depth of $8.9 \mathrm{~mm}$. Top: solution of the full wave equation. Middle: solution of the one-way wave equation. Bottom: difference between the two solutions. The line in the bottom picture indicates the location where the cross-section shown in Figure 8 is made. A $\pm 30 \mathrm{~dB}$ logarithmic grey scale is used in the display.

parabolic model has problems representing parts of the propagating pulse which travel at a wide angle out from the propagation axis.

Figure 7 shows that the pseudo-differential model is much more consistent with the pseudo-spectral solution of the full wave equation than the parabolic model was. To illustrate this further, a cross-section of the solutions is plotted in Figure 8. Not surprisingly, the pseudo-differential model is also able to resolve propagation at a wide angle out from the propagation axis. The discrepancy is caused by wave reflections of the propagating wave not being accurately accounted for in the one-way model.

A major benefit of using a one-way approximation to the wave equation, instead of the full wave equation, is the reduced computational complexity of finding a numerical solution. Although only a crude optimisation of the implementations was performed, the computational time for propagating a $5 \mathrm{MHz}$ pulse through the $9 \times 9 \mathrm{~mm}$ computational domain in Figure 5, using $256 \times 256$ spatial grid points and the same temporal sampling rate, was approximately four times longer for the full wave equation compared to the pseudo-differential method (4.5 min vs. $1.1 \mathrm{~min}$ on the available hardware; a $2.8 \mathrm{GHz}$ P4 running MATLAB 7 under Linux). Furthermore, the solution method based on one way wave equations scales linearly as a function of propagation distance. The advantage, therefore, increases for larger propagation distances. 

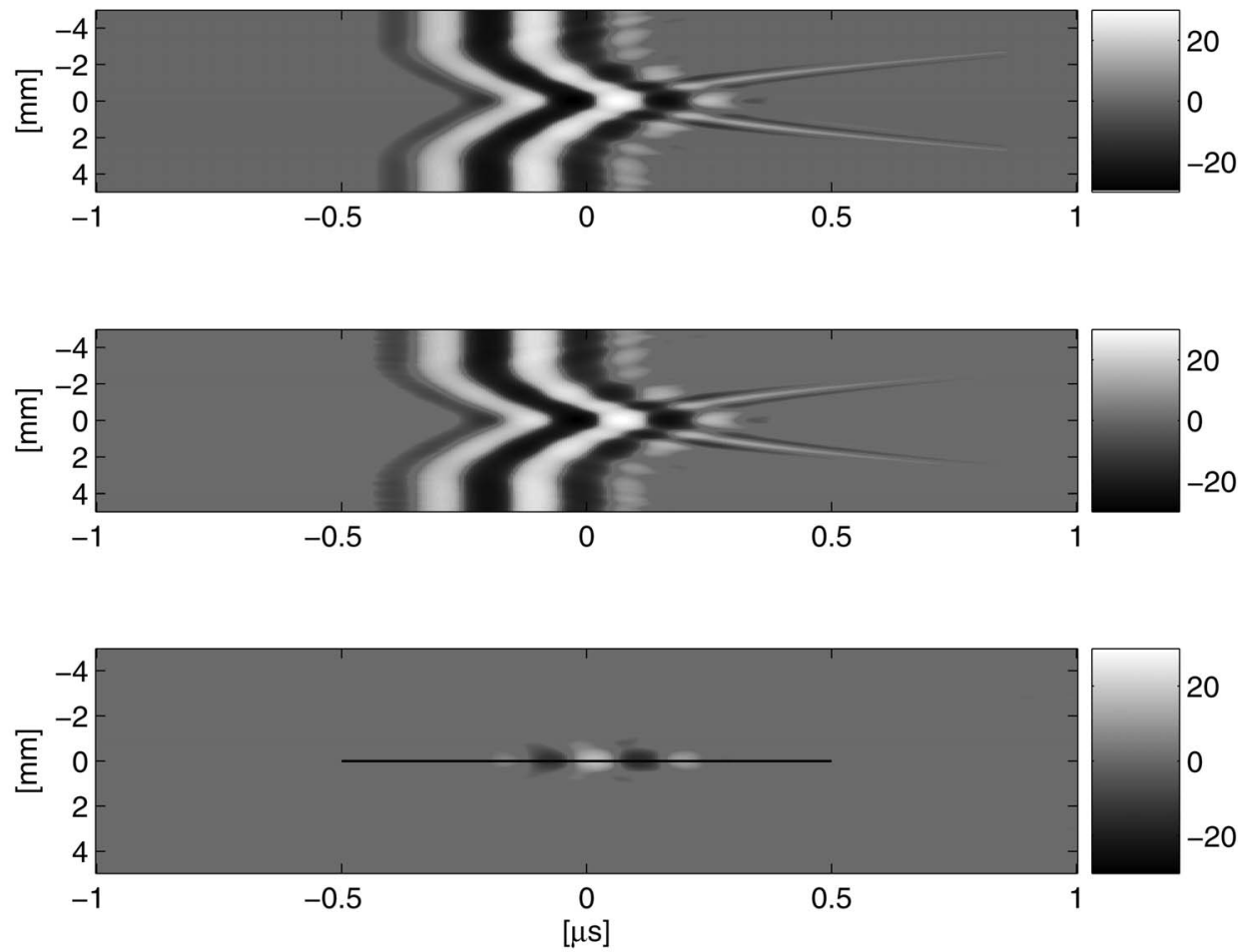

Figure 7. Comparison of the forward propagating wave fronts for the pseudo-differential model and the full wave equation recorded at $8.9 \mathrm{~mm}$. Top: solution of the full wave equation. Middle: solution of the one-way wave equation. Bottom: difference between the two solutions. The line in the bottom picture indicates the location where the crosssection shown in Figure 8 is made. A $\pm 30 \mathrm{~dB}$ logarithmic grey scale is used in the display.
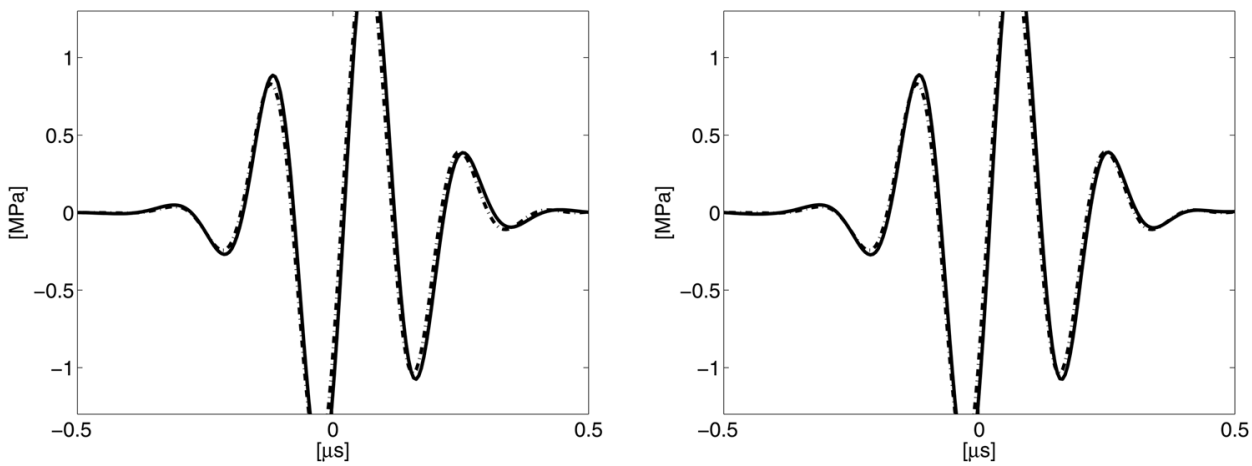

Figure 8. Cross-section comparing a numerical solution to the full wave equation (dash-dot line) to a numerical solution of the one-way models (solid line). Left: parabolic model. Right: pseudo-differential model.

\section{Parallelisation}

A major advantage of this scheme is the ease with which it is parallelised. Described here is a parallelisation for the pseudo-differential model, when $\rho$ is assumed constant. 
1. Distribute the solution for $z=0$ (i.e. $p_{0}(x, y, t)$ ) and medium parameters, $\varepsilon_{n}(x, y, z)$, $\varepsilon_{t}(x, y, z), \varepsilon(x, y, z)$ and $b(x, y, z)$, over $P$ processors in slices along the $x$-axis ranging from $x_{n}$ to $x_{n+1}$ on processor $n$.

2. Distribute propagation factor $U\left(k_{x}, k_{y}, \omega, h\right)$ over $P$ processors in slices along the $\omega$-axis ranging from $\omega_{n}$ to $\omega_{n+1}$ on processor $n$.

3. Apply $\mathcal{U}_{A_{n}}^{h}\left(z_{k}\right)$ and $\mathcal{U}_{A_{l}}^{h}\left(z_{k}\right)$ to the solution. This is performed locally at each processor, only requiring the pre-distributed material parameters.

4. Perform FFT along $y$ and $\omega$-axis. This is again performed locally on each processor yielding $p\left(x, k_{y}, \omega_{n} \ldots \omega_{n+1}\right)$.

5. Redistribute solution over $P$ processors in slices along the $\omega$-axis ranging from $\omega_{n}$ to $\omega_{n+1}$ on processor $n$.

6. Perform FFT along $x$-axis, yielding $p\left(k_{x}, k_{y}, \omega_{n} \ldots \omega_{n+1}\right)$ on processor $n$.

7. Multiply solution by $U\left(k_{x}, k_{y}, \omega, h\right)$. Again this only requires the pre-distributed propagation factor, and is thus a local operation.

8. Perform inverse FFT (IFFT) along $k_{x}$ and $k_{y}$, resulting in $p\left(x, y, \omega_{n} \ldots \omega_{n+1}\right)$.

9. Redistribute solution over $P$ processors in slices along the $x$-axis ranging from $x_{n}$ to $x_{n+1}$ on processor $n$.

10. Perform IFFT along $\omega$. The result on each processor now constitutes the solution one step of length $h$ forward.

11. Repeat steps 3 to 10 until the solution at a desired depth $z$ has been obtained.

It is worth pointing out that only steps 5 and 9 involve interprocessor communication. Furthermore, it is possible to subdivide the application of $u_{A_{n}}^{h}\left(z_{k}\right)$ and $u_{A_{l}}^{h}\left(z_{k}\right)$ into many smaller steps without additional communication. This may be required in order to avoid shock formation. Another nice feature is that the only essential difference between 2D and $3 \mathrm{D}$ is a Fourier transform along the $y$-axis. Thus a 3D implementation is possible based on a previous $2 \mathrm{D}$ implementation with only minor modifications.

Figure 9 shows spatial distribution of the acoustic energy when transmitting a $0.5 \mathrm{MPa}$ and $3 \mathrm{MHz}$ focused acoustic pulse from a $2 \times 2 \mathrm{~cm}$ square transducer through a homogeneous muscle-like tissue and through a heterogeneous tissue structure resembling the female breast. The simulation was performed using $256 \times 256 \times 512$ grid points for the computational domain which covered a $51.2 \times 51.2 \mathrm{~mm}$ xy-domain and a $7.3 \mu$ s time window. Propagating through an $80 \mathrm{~mm}$ domain in the z-direction took approximately 8 hours on a $2.8 \mathrm{GHz}$ P4 with 2 CPUs using the current MATLAB implementation (parallelisation with MPITB Baldomero (2005)).

The obtainable ultrasound image resolution is limited by the width of the transmitted beam. The width increases when propagating through the heterogeneous tissue. This spreading results in reduced peak-energy by about $5 \mathrm{~dB}$. The reduction has the effect of reducing the nonlinearly-generated energy by about $12 \mathrm{~dB}$. This reduction will in addition to reduced resolution due to a widening of the beam also result in a lower signal-to-noise ratio for the received back-scattered signal.

\section{Concluding remarks}

A one-way wave equation, Equation (9), for modelling the forward wave propagation of an ultrasound pressure field, along with a numerical solution method, has been presented. The equation was derived using a parabolic approximation to the full wave equation. The solution concurs with analytic reference solutions and experimental 

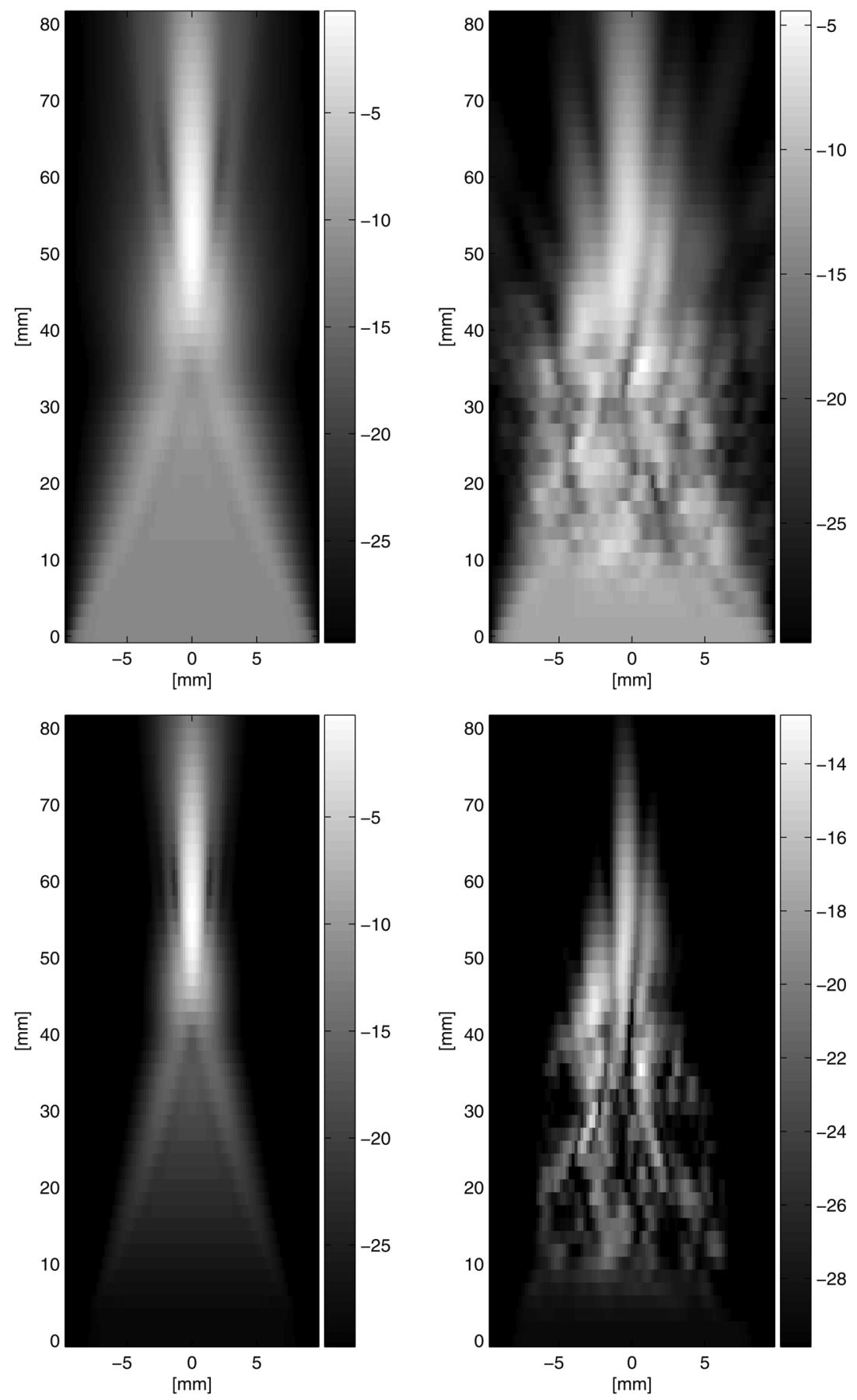

Figure 9. Acoustic energy distribution for when transmitting a $0.5 \mathrm{MPa}$ and $3 \mathrm{MHz}$ pulse from a $2 \times 2 \mathrm{~cm}$ square transducer focused at a depth of $6 \mathrm{~cm}$. Top left: total energy through homogeneous muscle-like tissue. Top right: total energy through heterogeneous tissue structure resembling female breast. Bottom left: nonlinearly generated energy in a band around $6 \mathrm{MHz}$ through homogeneous tissue. Bottom right: nonlinearly generated energy in a band around $6 \mathrm{MHz}$ through heterogeneous tissue. 
measurements in a homogeneous medium. The basic features of the numerical solution are also the same as those of a numerical reference, obtained as a solution to the full wave equation. However, for a deformed wave front, the propagation at large angles out from the axis of propagation is not accurately represented using the parabolic model.

In order to represent propagation in an aberrating medium, an approximation based on the angular spectrum method was applied. The resulting pseudo-differential model, while still being a one-way model, significantly improves the results over the parabolic model for the heterogeneous medium. When implemented using the FFT, the pseudodifferential model introduces periodic boundary conditions.

Thus, while being efficient and accurate, the boundary conditions introduce artifacts in the simulation. These were suppressed by applying a spatial window to the solution. A better solution may be to employ a higher-order (wide-angle) parabolic approximation which retains the accuracy at wide angles while facilitate PML boundary conditions.

The method has been parallelised in order to take advantage of multiple processors when solving larger 3D problems. The pseudo-differential model is particularly easy to distribute over a large number of processors. Extending a $2 \mathrm{D}$ code to perform $3 \mathrm{D}$ simulations was therefore relatively straight-forward.

\section{Acknowledgements}

The authors thank Safiye Dursun and Dr. Tonni Johansen for collection of the data used to validate the simulation. Dr. Gunnar Taraldsen is recognised for work on the 2D code. Professor Bjørn Angelsen and Professor Harald Krogstad at NTNU are thanked for useful discussions.

\section{References}

ANDERSON, M. E. (2000). "A 2D nonlinear wave propagation solver written in open-source matlab code," in Proc. 2000 IEEE-UFFC Ultrasonics Symposium. [Online]. Available: http:// www.seas.rochester.edu/maanders/sps2d.html

ANGELSEN, B. A. (2000). Ultrasound imaging. Waves, signals and signal processing. Trondheim, Norway: Emantec, vol. 2, http://www.ultrasoundbook.com.

BALDOMERO, J. F. (2005). "MPI toolbox for matlab,", http://atc.ugr.es/javier-bin/mpitb eng

Bamberg, A., Engqvist, B., Halpern, L. \& Joly, P. (1988). "Parabolic wave equation approximations in heterogeneous media," SIAM J. Appl. Math., vol. 48, no. 1, pp. 99-128.

BAmberG, A., ENGQVist, B., HALPERN, L. \& JOLY, P. (1988). "Higher order paraxial wave equation a approximations in heterogeneous media," SIAM J. Appl. Math., vol. 48, no. 1, pp. 129154.

BÉCACHe, E., Collino, F. \& Joly, P. (1998). "Higher-order numerical schemes and operator splitting for solving 3D paraxial wave equations in heterogeneous media," INRIA," Technical Report 3497.

BERENGER, J.-P. (1994). "A perfectly matched layer for the absorption of electromagnetic waves," J. Comp. Phys., vol. 114, no. 2, pp. 185-200.

BERKHOFF, A. P. \& THIJSSEN, J. M. (1996). "Correction of concentrated and distributed aberrations in medical ultrasound imaging," in Proc. 1996 IEEE-UFFC Ultrasonics Symposium, pp. 1405-1410.

Bouakaz, A., Lancée, C. \& De Jong, N. (2003). "Harmonic ultrasonic field of medical phased arrays: Simulations and measurements," IEEE Trans. Ultrason. Ferroelectr. Freq. Control, vol. 50, no. 6, pp. 730-735, June.

BREKHOVSKIKH, L. \& GODIN, O. (1999). Acoustics of Layered Media I-II, 2nd ed. Springer.

Christopher, P. T. \& PARKer, K. J. (1991). "New approaches to nonlinear diffractive fields propagation," J. Acoust. Soc. Am., vol. 90, no. 1, pp. 488-499, July. 
Claerbout, J. F. (1976). Fundamentals of Geophysical Data Processing. New York: McGrawHill.

Collino, F. (1997). "Perfectly matched absorbing layers for the paraxial equations," J. Comp. Phys., vol. 131, no. 1, pp. 164-180.

DollaRD, J. \& FRIEDMAN, C. (1979). Product Integration with Applications to Differential Equations, ser. Encyclopædia of mathematics and its applications, G.-C. Rota, Ed. Reading, Massachusetts: Addison-Wesley.

Duck, F. A. (1997). Physical properties of tissue. London: Academic Press.

Engl, H., Hanke, M. \& Neubauer, A. (2000). Regularization of inverse problems. Netherlands: Kluwer Academic Publishers.

Freiburger, P. D., Sullivan, D., LeBlanc, B. H. \& Trahey, G. E. (1992). "Two-dimensional ultrasonic beam distortion in the breast: in-vivo measurements and effects," Ultrason. Imaging, vol. 14, pp. 398-414.

Haberkorn, U., Layer, G., Rudat, V., Zuna, I., Lorenz, A. \& van Kaick, G. (1993). "Ultrasound image properties influenced by abdominal wall thickness and composition," $J$. Clin. Ultrasound, vol. 21, pp. 423-429.

Hamilton, M. F. \& Blackstock, D. T. (1997). Nonlinear Acoustics. San Diego: Academic Press.

Hinkelman, L., Liu, D.-L., Metlay, L. A. \& WaAg, R. C. (1994). "Measurements of ultrasonic pulse arrival time and energy level variations produced by propagation through abdominal wall," J. Acoust. Soc. Am., vol. 95, no. 1, pp. 530-541, January.

Holden, H. \& Risebro, N. H. (2002). Front Tracking for Hyperbolic Conservation Laws, ser. Applied Mathematical Sciences. New York: Springer Verlag, vol. 152.

KapPel, I. (1997). Evolution equations and Approximations. San Diego: Academic Press.

Kuznetsov, V. P. (1971). "Equations of nonlinear acoustics," Sov. Phys. Acoust., vol. 16, pp. 467-470.

Måsøy, S. E., Johansen, T. F. \& Angelsen, B. (2003). "Correction of ultrasonic wave aberration with a time delay and amplitude filter," J. Acoust. Soc. Am., vol. 113, no. 4, pp. 2009-2020, April.

MAST, T. D. (2002). "Two- and three-dimensional simulations of ultrasonic propagation through human breast tissue," Acoust. Res. Lett. Online, vol. 2, no. 3, pp. 53-58, April.

Mast, T. D., Hinkelman, L., OrR, M. \& WaAG, R. C. (1998). "The effect of abdominal wall morphology on ultrasonic pulse distortion. part II. simulations," J. Acoust. Soc. Am., vol. 104, no. 6, pp. 3651-3664, December.

Nachman, A. I., Smith, J. F. \& WAaG, R. C. (1990). "An equation for acoustic propagation in inhomogeneous media with relaxation losses," J. Acoust. Soc. Am., vol. 88, pp. 1584-1595.

StRAng, G. (1968). "On the construction and comparison of difference schemes," Numer. Anal., vol. 5, pp. 506-517.

SzABO, T. L. (1994). "Time domain wave equations for lossy media obeying a frequency power law," J. Acoust. Soc. Am., vol. 96, pp. 491-500.

TABeI, M., Mast, T. D. \& WAAG, R. C. (2003). "Simulation of ultrasonic focus aberration and correction through human tissue," J. Acoust. Soc. Am., vol. 113, no. 2, pp. 1166-1176, February.

TAPPERT, F. (1977). "The parabolic approximation method in wave propagation and underwater acoustics," in Lectures in Physics, J. B. Keller and J. S. Papadakis, Eds. New York: Springer, pp. 224-287.

TARALDSEN, G. (2001). "Derivation of a generalized Westervelt equation for nonlinear medical ultrasound," J. Acoust. Soc. Am., vol. 109, no. 4, pp. 1329-1333, April.

TraheY, G. E., Freiburger, P. D., Nock, L. F. \& Sullivan, D. C. (1991). "In-vivo measurements of ultrasonic beam distortion in the breast," Ultrason. Imaging, vol. 13, no. 1, pp. 71-90.

VARSLOT, T. \& TARALDSEN, G. (2005). "Computer simulation of forward wave propagation in soft tissue," IEEE Trans. Ultrason. Ferroelectr. Freq. Control, vol. 52, no. 9, pp. 1473-1782.

VARSLOT, T. (1999). "Wavefront aberration in medical ultrasound imaging," Dr.ing. thesis, Institutt for matematiske fag, NTNU, Trondheim, 2004.

Whitham, G. B. (1974). Linear and Nonlinear Waves. Wiley.

Wojcik, G. L., Fornberg, B., WaAg, R. C., Carcione, L., Mould, J., Nikodym, L. \& Driscoll, T. (1997). "Pseudospectral methods for large-scale bioacoustic models," in Proc. 1997 IEEE-UFFC Ultrasonics Symposium, pp. 1501-1506. 
Wojcik, G., Mould, J., Ayter, F. \& Carcione, L. (1998). "A study of second harmonic generation by focused medical transducer pulses," in Proc. 1998 IEEE-UFFC Ultrasonics Symposium, pp. 1583-1588.

ZaвотSKAYA, E. A. \& KhOKLOv, R. V. (1969). "Quasi-plane waves in the non-linear acoustics of confined beams," Sov. Phys. Acoust., vol. 15, pp. 35-40.

Zhu, Q. \& Steinberg, B. D. (1994). "Wavefront amplitude distribution in the female breast," $J$. Acoust. Soc. Am., vol. 96, no. 1, pp. 1-9, April. 